\title{
Non-immersion theorems for lens spaces
}

By

Teiichi Kobayashi

(Received September 28, 1966)

\section{\$1. Introduction}

The purpose of this paper is to show some non-immersion theorems for lens spaces. For the proof we shall use the theorem of $\mathrm{T}$. Kambe which determines the structure of $K_{\mathrm{A}}$-rings of the lens space [6] and the theorem of T. Kambe, H. Matsunaga and H. Toda on stunted lens spaces [7].

Throughout this note $p$ is always an odd prime. Let $S^{2 n+1}$ be the unit $(2 n+1)$-sphere. A point of $S^{2 n+1}$ is represented by a sequence $\left(z_{0}, \cdots, z_{n}\right)$ of complex numbers $z_{i}(i=0, \cdots, n)$ with $\sum\left|z_{i}\right|^{2}=1$. Let $\gamma$ be the rotation of $S^{2 n+3}$ defined by

$$
\gamma\left(z_{0}, \cdots, z_{n}\right)=\left(\lambda z_{0}, \cdots, \lambda z_{n}\right), \quad \text { where } \lambda=e^{2 z_{i} / p},
$$

and let $\Gamma$ be the topological transformation group of $S^{2 n+1}$ of order $p$ generated by $\gamma$. Then

$$
L^{n}(p)=S^{2 n+1} / \Gamma
$$

is the lens space $\bmod p$. It is an $(2 n+1)$-dimensional compact, connected differentiable manifold without boundary. Let $\left\{z_{0}, \cdots, z_{n}\right\}$ $\varepsilon L^{n}(p)$ denote the equivalence class of $\left(z_{0}, \cdots, z_{n}\right) \in S^{2 n+1}$. The space $L^{k-1}(p)$ is naturally embedded in $L^{k}(p)$ by identifying $\left\{z_{0}, \cdots, z_{k-1}\right\}$ with $\left\{z_{0}, \cdots, z_{k-1}, 0\right\}$. Let $L_{0}^{k}(p)=\left\{\left\{z_{0}, \cdots, z_{k}\right\} \in\right.$ $L^{k}(p) \mid z_{k}$ is real and $\left.z_{k} \geqq 0\right\}$. Then $L^{k}(p)-L_{0}^{k}(p)=e^{2 k+1}((2 k+1)$-cell $)$ and $L_{0}^{k}(p)-L^{k-1}(p)=e^{2 k}(2 k-$ cell $), k \leqq n$. Thus $L^{n}(p)$ has a cell structure given by

$$
L^{n}(p)=S^{1} \cup e^{2} \cup e^{3} \cup \cdots \cup e^{2 n} \cup e^{2 n+1} .
$$

(cf. [6] and [7]). 
Let $M^{n}$ be an $n$-dimensional differentiable manifold and $R^{*}$ be the $k$-dimensional Euclidean space. By $M^{n} \subseteq R^{k}$ (respectively $M^{n} \mp R^{k}$ ) we mean that $M^{n}$ can be immersed (respectively cannot be immersed) in $R^{k}$.

After some preparations in $\$ 2$, we shall give in $\$ 3$ a neces. sary condition for immersibility of certain lens spaces (Theorem 3). As applications, in $\$ 4$ we shall prove some non-immersion theorems for lens spaces. For example, we obtain the following result (Theorem 4. $(I I)$ ).

Let $p$ be a prime with $p \geqq 5$. Let $\alpha$ and $\beta$ be odd integers such that $0<\alpha \leqq(2 p-1) / 3$ and $0<\beta \leqq(p-2) / 3$, and let $l$ and $k$ be integers such that $l>k \geqq 0$ and $l>1$ if $\alpha>1$, or $l>k \geqq 0$ and $l>2$ if $\alpha=1$.

Then $L^{n}(p) \mp R^{3 n+1}$ for $n=\alpha p^{l}+\beta p^{k}$.

The method of the proof is similar to that of J. Adem and S. Gitler [1] with which they have given a simple proof for the James' non-immersion theorem for real projective spaces ([5], Theorem 1.1). In [1], they uses the twisted normal bundle and the S-reducibility, while we shall use the ordinary normal bundle and the Steenrod reduced power operations.

I thank Professors A. Komatsu and H. Toda for valuable suggestions, and also Professors M. Sugawara and Y. Saito for kind advice.

\section{§2. Preliminaries}

Let $X$ be a finite connected $C W$-complex. Let $\varepsilon(X)$ denote the set of equivalence classes of real vector bundles over $X$ and let

$$
\theta: \varepsilon(X) \rightarrow K O(X)
$$

be a natural map, where $K O(X)$ is the associated Grothendieck group. When we consider the complex vector bundles, the associated Grothendieck group is denoted by $K(X)$.

An element $\alpha \in K O(X)$ is said to be positive if there is an element $\beta \in \varepsilon(X)$ such that $\theta(\beta)=\alpha$. We shall drop the symbol $\theta$ and regard (an equivalence class of) a vector bundle as an element 
of both $\varepsilon(X)$ and $K O(X)$.

A geometrical dimension of an element $\alpha \in K O(X)$ (written $g \cdot \operatorname{dim} \alpha$ ) is the least integer $k$ such that $\alpha+k$ is positive, where $k$ is the $k$-dimensional trivial bundle over $X$.

Let $C P^{n}$ be the complex projective space of complex $n$ dimension. Let $\xi\left(\in K\left(C P^{n}\right)\right)$ denote the canonical line bundle over $C P^{n}$ and $r(\xi)\left(\in K O\left(C P^{n}\right)\right)$ denote the real restriction of $\xi$. Let

$$
\pi: L^{n}(p) \rightarrow C P^{n}
$$

be the natural projection. Define

$$
\eta=\pi^{*} r(\xi)=r\left(\pi^{*} \xi\right) \in K O\left(L^{n}(p)\right),
$$

that is, $\eta$ is the induced bundle of $r(\xi)$ by $\pi$.

Let $\tau$ be the tangent bundle of $L^{n}(p)$. Then the following equality holds (cf. [6], Lemma $(4,7)$ ), where $\oplus$ denotes the Whitney sum.

$$
\tau \oplus 1=(n+1) \eta .
$$

Define $\bar{\sigma}=\eta-2\left(\in \widetilde{K O}\left(L^{n}(p)\right)\right.$, the stable class of $\eta$. The main theorem of T. Kambe ([6], Theorem 2) is as follows.

Theorem. Let $p$ be an odd prime, $q=(p-1) / 2$ and $n=s(p-1)$ $+r(0 \leqq r<p-1)$. Then

$$
\widetilde{K O}\left(L^{n}(p)\right) \approx \begin{cases}\left(Z_{p^{s+1}}\right)^{[r / 2]}+\left(Z_{p^{s}}\right)^{q-\left[r^{r / 2]}\right.} & \text { (if } n \neq 0 \bmod 4) \\ Z_{2}+\left(Z_{p^{s+1}}\right)^{[r / 2]}+\left(Z_{p^{s}}\right)^{r-[r / 2]} & \text { (if } n \equiv 0 \bmod 4)\end{cases}
$$

and the direct summand $\left(Z_{p^{s+1}}\right)^{[r / 2]}$ and $\left(Z_{p^{s}}\right)^{q-[r / 2]}$ are generated additively by $\bar{\sigma}, \cdots, \bar{\sigma}^{[r / 2]}$ and $\bar{\sigma}^{\left[r^{2 / 2]+1}\right.}, \cdots, \sigma^{q}$ respectively. Moreover its ring structure is given by

$$
\bar{\sigma}^{g+1}=\sum_{i=1}^{q} \frac{-(2 q+1)}{2 i-1}\left(\begin{array}{c}
q+i-1 \\
2 i-2
\end{array}\right) \bar{\sigma}^{i}, \bar{\sigma}^{[n / 2]+1}=0 .
$$

In the theorem, $\left(Z_{a}\right)^{b}$ indicates the direct sum of $b$-copies of a cyclic group $Z_{a}$ of order $a$ and $[c]$ denotes the integral part of $c$. Note that

$$
p^{s+\frac{a}{a}}=0,
$$

where $\varepsilon=0$ or 1 according as $[r / 2]=0$ or $[r / 2] \geqq 1$. 
Then we can prove the following theorem.

Theorem 1. Let $n$ and $k$ be positive integers with $0<k \leqq 2 n+1$ and let $a$ be $a$ positive integer such that $2 a p^{s+2}>4 n+3$. The necessary and sufficient condition for $L^{n}(p) \subseteq R^{2 n+1+k}$ is that the bundle $\left\{a p^{s+\varepsilon}-(n+1)\right\} \eta$ has $\left\{2 a p^{s+\varepsilon}-(2 n+k+2)\right\}$ independent nonzero cross-sections.

Proof. If $L^{n}(p)$ is immersed in $R^{2 n+1+k}$, then we have a normal bundle $\nu$ of dimension $k$ and

$$
\tau \oplus v=2 n+k+1
$$

Hence, by (1), (2) and (3), we have

$$
\nu-k=\left\{a p^{s+\varepsilon}-(n+1)\right\} \eta-2\left\{a p^{s+q}-(n+1)\right\} \in \widetilde{K O}\left(L^{n}(p)\right) .
$$

Thus we see

$$
\nu \oplus\left\{2 a p^{s+2}-(2 n+k+2)\right\}=\left\{a p^{s+1}-(n+1)\right\} \eta,
$$

since the dimension of the bundles of both sides is greater than $2 n+1$ (cf. [10], Lemma (1.2)). The formula (4) implies that the bundle $\left\{a p^{s+z}-(n+1)\right\} \eta$ has $2 a p^{s+z}-(2 n+k+2)$ inpependent nonzero cross-sections.

Assume that there exists a vector bundle $\alpha$ of dimension $k$ such that

$$
\left\{a p^{s+q}-(n+1)\right\} \eta=\alpha \oplus\left\{2 a p^{s+?}-(2 n+k+2)\right\} .
$$

Denote by $\tau_{0}$ the stable class of $\tau$. From (1), (2) and (5) we have $-\tau_{0}=\alpha-k$, and so $g \cdot \operatorname{dim}\left(-\tau_{0}\right) \leqq k$. Therefore, by the theorem of Hirsch ([4], Theorem 6.4 and [3], Proposition 3.2) we have

$$
L^{n}(p) \subseteq R^{2 n+1+\star} \text {. }
$$

The cohomology algebra over $Z_{p}$ of $L^{\prime \prime}(p)$ is given as follows (cf. [11], p. 68).

$H^{*}\left(L^{n}(p) ; Z_{p}\right)$ is the tensor product of the exterior algebra on a generator $y \in H^{1}\left(L^{n}(p) ; Z_{p}\right)$ and the truncated polynomial algebra on a generator $x \in H^{2}\left(L^{n}(p) ; Z_{p}\right)$ with relations $y^{2}=0, \Delta y=-x$ and $x^{n+1}=0$, where $\Delta$ is the Bockstein coboundary operator associated with the exact coefficient sequence: $0 \rightarrow Z_{p} \rightarrow Z_{p} \rightarrow Z_{p} \rightarrow 0$. 


\section{$\$ 3$. Stunted lens spaces}

Let $\alpha$ be a vector bundle over $X$ and let $X^{*}$ denote its Thom complex. For a positive integer $t$ and a space $Y$, denote by $S^{t} Y$ the $t$-fold suspension of $Y$. The following result is shown by M. F. Ativah ([2], Lemma (2.4)).

There is a natural homeomorphism:

$$
S^{t}\left(X^{\infty}\right) \approx X^{* \oplus t} .
$$

Recently. T. Kambe, H. Matsunaga and H. Toda have proved the following theorem on stunted lens spaces ([7]. Theorem 1).

There exists a natural homeomorphism:

$$
L^{m}(p) / L^{m-n-z}(p) \approx\left(L^{n}(p)\right)^{(m-n) n} .
$$

By making use of these theorems we have the following result.

Theorem 2. Let $n$ and $k$ be positive integers with $0<k \leqq 2 n+1$, and let $n=s(p-1)+r(0 \leqq r<p-1)$. Assume that $a$ is a positive integer such that $2 a p^{s+e}>4 n+3$ and that $t=2 a p^{s+8}-(2 n+k+2)$, where $\varepsilon=0$ or 1 according as $[r / 2]=0$ or $[r / 2] \geqq 1$. If $L^{n}(p)$ is immersed in $R^{2 n+1+k}$ with a normal bundle $\nu$, then there exists a natural homeomorphism:

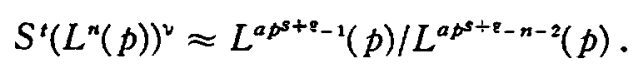

Proof. As in the proof of Theorem 1, we see

$$
\nu \oplus t=\left\{a p^{s-s}-(n+1)\right\} \eta \text {. }
$$

Then we have

$$
\begin{aligned}
& \left.S^{f}\left(L^{n}(p)\right)^{\nu} \approx\left(L^{n}(p)\right)\right)^{\bullet t}=\left(L^{n}(p)\right)^{\left\{a p^{\left.s+\varepsilon_{-}(n+1)\right\} \eta}\right.} \\
& \approx L^{a p^{s+z_{-1}}}(p) / L^{a p s+\varepsilon_{-n-2}}(p) .
\end{aligned}
$$

Let $\alpha=(E, p, X)$ be an oriented vector bundle of dimension $k$ with the total space $E$, the base space $X$ and the projection $p: E \rightarrow X$. Here, we assume that the base space $X$ is a finite connected $C W$-complex. Denote by $E_{0}$ the subspace of $E$ which consists of non-zero vectors. Then the following diagram is commutative (cf. [12]). 


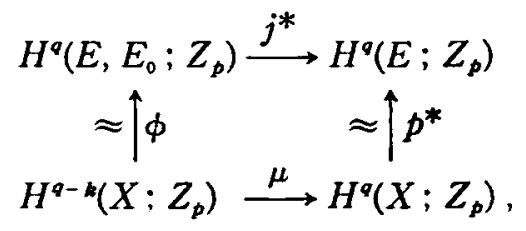

where $j^{*}$ is a map induced by the injection, $\phi$ is the Thom isomorphism and $\mu$ is defined by

$$
\mu(y)=y \cdot \chi \quad \text { for } \quad y \in H^{q-h}\left(X ; Z_{p}\right),
$$

where $\chi$ is the $\bmod p$ Euler class of $\alpha$.

If $\mu$ is an isomorphism, so is $j^{*}$. Therefore, if $\lambda: X \rightarrow X^{*}$ is the inclusion map induced by the zero cross-section, then the induced map

$$
\lambda^{*}: H^{q}\left(X^{a} ; Z_{p}\right) \rightarrow H^{q}\left(X ; Z_{p}\right) \quad \text { for } \quad k \leqq q \leqq \operatorname{dim} X
$$

is also an isomorphism.

Theorem 3. Let $n$ and $l$ be positive integers with $0<l \leqq n$, and let $n=s(p-1)+r(0 \leqq r<p-1)$. Assume that $a$ is a positive integer such that $2 a p^{s+p}>4 n+3$ and that $t=2 a p^{s+2}-2(n+l+1)$, where $\varepsilon=0$ or 1 according as $[r / 2]=0$ or $[r / 2] \geqq 1$. If $L^{n}(p)$ is immersed in $R^{2 n+1+2 l}$ with a normal bundle $\nu$ whose Euler class is non-zero, then there is a map

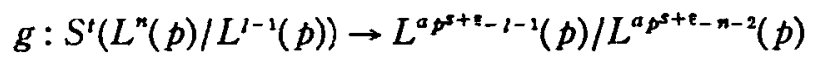

which induces isomorphisms of all cohomology groups with $Z_{p}$ coefficients.

Proof. Since the $\bmod p$ reduction induces an isomorphism :

$$
H^{2 \prime}\left(L^{n}(p) ; Z\right) \approx H^{2 \prime}\left(L^{\prime \prime}(p) ; Z_{p}\right),
$$

the $\bmod p$ Euler class $\bar{\chi}$ of $\nu$ is non-zero. The group $H^{2}\left(L^{n}(p) ; Z_{p}\right)$ $\left(=Z_{p}\right)$ is generated by $x^{\prime}$, where $x$ is a generator of $H^{2}\left(L^{n}(p) ; Z_{p}\right)$. Hence, $\bar{\chi}=m x^{l}$ for some $m$ with $0<m<p$, and so we have an isomorphism :

$$
\mu=\bar{\chi}: H^{q-2 l}\left(L^{n}(p) ; Z_{p}\right) \approx H^{q}\left(L^{n}(p) ; Z_{p}\right) \quad \text { for } \quad 2 l \leqq q \leqq 2 n+1 .
$$

Therefore, if $\lambda: L^{n}(p) \rightarrow\left(L^{n}(p)\right)^{\nu}$ is the natural inclusion, $\lambda$ induces 
an isomorphism :

$$
\lambda^{*}: H^{q}\left(\left(L^{n}(p)\right)^{x} ; Z_{p}\right) \approx H^{q}\left(L^{n}(p) ; Z_{p}\right) \quad \text { for } \quad 2 l \leqq q \leqq 2 n+1 .
$$

Since $\left(L^{n}(p)^{3}\right.$ is $(2 l-1)$-connected, there is a map $f$ such that the following diagram is homotopy-commutative, where $q: L^{n}(p) \rightarrow$ $L^{n}(p) / L^{l-1}(p)$ is the projection.

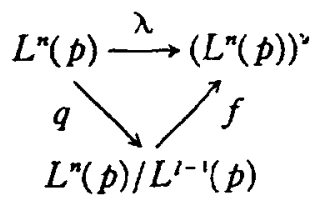

It is easily verified that the map $f$ induces an isomorphism: $\left.f^{*}: H^{q}\left(L^{n}(p)\right)^{\nu} ; Z_{p}\right) \approx H^{q}\left(L^{n}(p) / L^{1-1}(p) ; Z_{p}\right)$ for $0 \leqq q \leqq 2 n+1$.

Let $S^{t} f$ be the $t$-fold suspension of the map $f$ and let

$$
\phi: S^{t}\left(L^{n}(p)\right)^{\nu}=L^{a p s+z_{-1}}(p) / L^{a p^{s+2-n-2}(p)}
$$

be a homeomorphism given in Theorem 2. Since the complex $S^{t}\left(L^{n}(p) / L^{1-1}(p)\right)$ has dimension $2 a p^{s+e}-2 l-1$, the image of a cellular approximation to the map $\varphi \circ S^{t} f$ is contained in the $\left(2 a p^{s+q}-2 l-1\right)$-dimensional skeleton of $L^{a p s+q-1}(p) / L^{a p s+q-n-2}(p)$. Thus there exists a map $g$ such that the following diagram is homotopy-commutative, where $i$ is the inclusion.

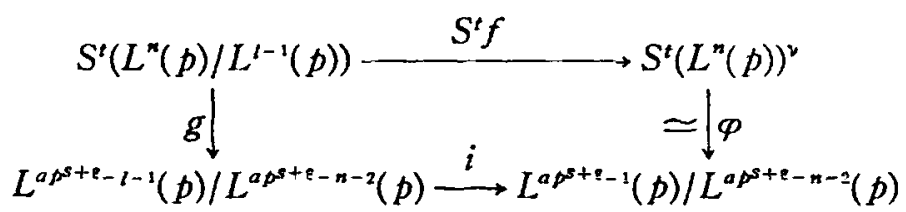

Then we can see that the map $g$ induces isomorphisms of all cohomology groups with $Z_{p}$ coefficients.

\section{$\$ 4 . \quad$ Applications}

In this section we apply Theorem 3 to get some non-immersion theorems for lens spaces. First, we recall the Pontrjagin class $\bmod p$ of lens spaces $L^{n}(p)$ (cf. [9]).

Let $x \in H^{2}\left(L^{n}(p) ; Z_{p}\right)$ be a generator. The total Pontrjagin 
class $\bmod p$ of $L^{n}(p)$ is given by the equation :

$$
p\left(L^{n}(p)\right)=\left(1+x^{2}\right)^{n+1},
$$

and the dual Pontrjagin class $\bmod p$ is given by the equation:

$$
\bar{p}\left(L^{n}(p)\right)=\left(1+x^{2}\right)^{-n-1}=\sum_{i=0}^{n / 23}(-1)^{i}\left(\begin{array}{c}
n+i \\
i
\end{array}\right) x^{2 i} .
$$

Theorem 4. Let $p$ be a prime with $p \geqq 5$. Assume that either of the conditions $(I)$ and $(I I)$ below is satisfied.

(I) $\alpha$ and $\beta$ are even integers such that $0 \leqq \alpha \leqq(2 p-2) / 3$ and $0<\beta \leqq(2 p-2) / 3$, and $l$ and $k$ are integers such that $l>k \geqq 0$ and $l>1$ if $\alpha>0$, or $k>1$ if $\alpha=0$.

(II) $\alpha$ and $\beta$ are odd integers such that $0<\alpha \leqq(2 p-1) / 3$ and $0<\beta \leqq(p-2) / 3$, and $l$ and $k$ are integers such that $l>k \geqq 0$ and $l>1$ if $\alpha>1$, or $l>k \geqq 0$ and $l>2$ if $\alpha=1$.

Then $L^{n}(p) \nsubseteq R^{3 n+1}$ for $n=\alpha p^{l}+\beta p^{k}$.

Proof. First, we consider the case when the condition $(I)$ is satisfied. Suppose that $L^{n}(p) \subseteq R^{3 n+1}$ for $n=\alpha p^{j}+\beta p^{k}$. Let $\nu$ be an oriented normal vector bundle of dimension $n$. The highest dimensional non-zero Pontrjagin class $\bmod p$ of $\nu$ is

$$
\bar{p}_{n / 2}=(-1)^{n / 2}\left(\begin{array}{c}
n+n / 2 \\
n / 2
\end{array}\right) x^{n}\left(\in H^{2 n}\left(L^{n}(p) ; Z_{p}\right)\right)
$$

where $x$ is a generator of $H^{2}\left(L^{n}(p) ; Z_{p}\right)$, since

$$
\left(\begin{array}{c}
n+n / 2 \\
n / 2
\end{array}\right)=\left(\begin{array}{c}
\frac{3 \alpha}{2} p^{\prime}+\frac{3 \beta}{2} p^{k} \\
\frac{\alpha}{2} p^{\prime}+\frac{\beta}{2} p^{k}
\end{array}\right) \equiv\left(\begin{array}{c}
\frac{3 \alpha}{2} \\
\frac{\alpha}{2}
\end{array}\right) \cdot\left(\begin{array}{c}
\frac{3 \beta}{2} \\
\frac{\beta}{2}
\end{array}\right) \neq 0(\bmod p)^{1)}
$$

Let $\bar{\chi}\left(\in H^{n}\left(L^{n}(p) ; Z_{p}\right)\right)$ be the Euler class $\bmod p$ of $\nu$. It is well known (e.g., [8]. Theorem 31) that

$$
\bar{\chi}^{2}=\bar{p}_{n / 2} \text {. }
$$

1) If $\mathrm{a}=\sum_{i} a_{i} p^{i}$ and $b=\sum_{i} b_{i} p^{i}$ are $p$-adic expansions, then $\left(\begin{array}{l}a \\ b\end{array}\right) \equiv \operatorname{II}_{i}\left(\begin{array}{l}a_{i} \\ b_{i}\end{array}\right) \quad(\bmod p)$. 
Thus we have $\bar{\chi} \neq 0$. Let $s$ and $r$ be integers given by the equation :

$$
\alpha p^{l}+\beta p^{k}=s(p-1)+r \quad(0 \leqq r<p-1),
$$

and let $v(=s+\varepsilon)$ be $s$ or $s+1$ according as $[r / 2]=0$ or $[r / 2] \geqq 1$ respectively. Denote by $a$ an integer with $2 a p^{n}>4 n+3$.

Now, by Theorem 3 , there exists a map

$$
g: S^{t}\left(L^{n}(p) / L^{(n-2) / 2}(p)\right) \rightarrow L^{a p^{y-}-(n+2) / 2}(p) / L^{a p^{\nu-n-2}(p)}
$$

which induces isomorphisms of all cohomology groups with $Z_{p}$ coefficients, where $t$ is a positive integer given by

$$
t=2 a p^{v}-3 n-2 \text {. }
$$

Let

$$
E^{t}: H^{q-t}\left(L^{n}(p) / L^{(n-2) / 2}(p) ; Z_{p}\right) \approx H^{q}\left(S^{t}\left(L^{n}(p) / L^{(n-2) / 2}(p)\right) ; Z_{p}\right)
$$

be the $t$-fold suspension isomorphism and let $\left(E^{t}\right)^{-1} \circ g^{*}=G$. Since $E$ and $g^{*}$ commute with Steenrod reduced power operations respectively, so is $G$.

Define a positive integer $q$ by the equation:

$$
q=2 a p^{0}-n-2 p^{2}
$$

and consider the following commutative diagram, where the two $\rho^{1}$ are first Steenrod reduced power operations $\bmod p$.

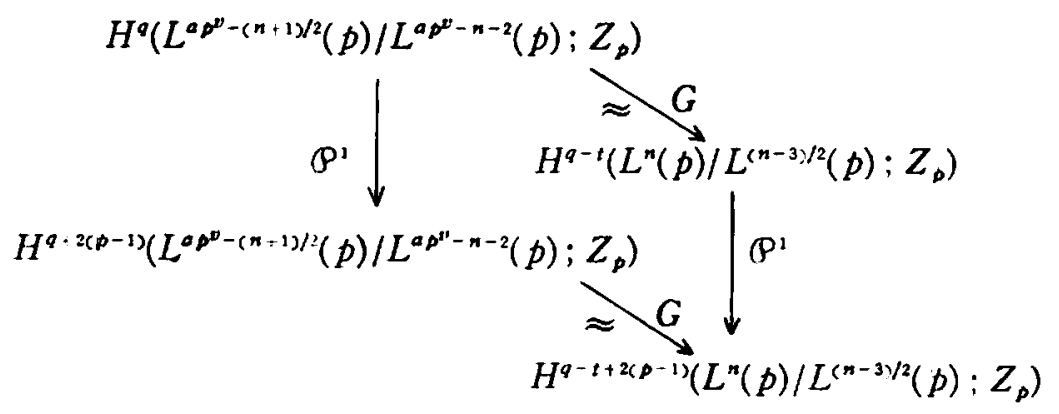

It is easily seen that each group in the diagram is non-zero. Note that

$$
q-\left\{2\left(a p^{v}-n-2\right)+1\right\}=\alpha p^{l}+\beta p^{k}-2 p^{2}+3 \geqq 3
$$

by the assumption. Therefore, the two operations $\rho^{1}$ on the left 
and right in the diagram are equivalent to the following two operations respectively :

$$
\begin{aligned}
& \rho^{1}: H^{q}\left(L^{a p^{p}-(n+2) / 2}(p) ; Z_{p}\right) \rightarrow H^{q+2(p-1)}\left(L^{a p^{p}-(x+2) / 2}(p) ; Z_{p}\right), \\
& \rho^{i}: H^{q-t}\left(L^{n}(p) ; Z_{p}\right) \rightarrow H^{q-t+2(p-1)}\left(L^{n}(p) ; Z_{p}\right) .
\end{aligned}
$$

Let $k>0$. For a generator $x^{q / 2}$ of $H^{q}\left(L^{a p^{\nu}-(n+2) / 2}(p) ; Z_{p}\right)$ we have $P^{1} x^{q / 2}=0$, since $q / 2 \equiv 0(\bmod p)^{2}$. On the other hand, for a generator $x^{(q-t) / 2}$ of $H^{q-t}\left(L^{n}(p) ; Z_{p}\right)$ we have $\mathcal{P}^{1} x^{(q-t) / 2} \neq 0$, since $(q-t) / 2 \equiv 0(\bmod p)$.

Therefore, in the diagram, $\beta^{1}$ on the left is trivial, while $\beta^{1}$ on the right is non-trivial. This is a contradiction.

If $k=0$, consider the following commutative diagram, where the two $P^{p}$ are $p$-th reduced power operations $\bmod p$.

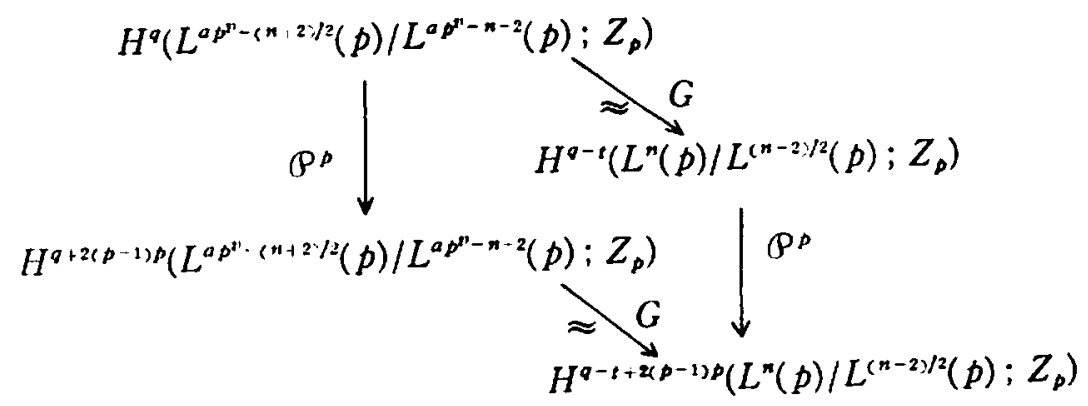

As is easily seen, each group in the diagram is non-zero. Note that

$$
q-\left\{2\left(a p^{n}-n-2\right)+1\right\}=\alpha p^{l}+\beta-2 p^{2}+3>3
$$

by the assumption. Thus we carry the proof as in the above case.

For a generator $x^{q / 2}$ of $H^{q}\left(L^{a \rho^{\theta}-(x+2) / 2}(p) ; Z_{p}\right)$ we have

$$
\mathcal{P}^{p} x^{q / 2}=\left(\begin{array}{c}
q / 2 \\
p
\end{array}\right) x^{q / 2+p-1) p}=-x^{q / 2+(p-1) p} \neq 0,
$$

since $q / 2=(a-1) p^{p}+(p-1) p^{r-1}+\cdots+(p-1) p^{l+1}+(p-1-\alpha / 2) p^{l}$ $+(p-1) p^{l-1}+\cdots+(p-1) p^{3}+(p-2) p^{2}+(p-1) p+(p-\beta / 2)$. On the other hand, for a generator $x^{\langle q-t / 2}$ of $H^{q-t}\left(L^{n}(p) ; Z_{p}\right)$ we have

2) $\mathcal{P}^{r} x^{i}=\left(\begin{array}{l}i \\ r\end{array}\right) x^{i+(p-1) r}$. 


$$
\rho^{p} x^{(q-t) / 2}=\left(\begin{array}{c}
(q-t) / 2 \\
p
\end{array}\right) x^{(q-t) / 2+(p-1) p}=0
$$

since $(q-t) / 2=(\alpha-1) p^{i}+(p-1) p^{l^{-1}}+\cdots+(p-1) p^{2}+(\beta+1)$.

Therefore, in the diagram, $\beta^{p}$ on the left is non-trivial, while $\mathcal{Q}^{p}$ on the right is trivial. This is a contradiction.

Next, we consider the case when the condition $(I I)$ is satisfied. Suppose that $L^{n}(p) \subseteq R^{3 n+1}$ for $n=\alpha p^{i}+\beta p^{k}$. Let $\nu$ be an oriented normal bundle of dimension $n$. The highest dimensional non-zero Pontrjagin class $\bmod p$ of $\nu$ is $\bar{p}_{n / 2}\left(\in H^{2 n}\left(L^{n}(p) ; Z_{p}\right)\right.$ ), because

$$
\begin{aligned}
\left(\begin{array}{c}
n+n / 2 \\
n / 2
\end{array}\right) & =\left(\begin{array}{l}
\frac{3 \alpha-1}{2} p^{\prime}+\frac{p-1}{2} p^{i-2}+\cdots+\frac{p-1}{2} p^{k+1}+\frac{p+3 \beta}{2} p^{k} \\
\frac{\alpha-1}{2} p^{\prime}+\frac{p-1}{2} p^{\prime-}+\cdots+\frac{p-1}{2} p^{k+1}+\frac{p+\beta}{2} p^{k}
\end{array}\right) \\
& \equiv\left(\begin{array}{c}
\frac{3 \alpha-1}{2} \\
\frac{\alpha-1}{2}
\end{array}\right) \cdot\left(\begin{array}{c}
\frac{p+3 \beta}{2} \\
\frac{p+\beta}{2}
\end{array}\right) \neq 0(\bmod p) .
\end{aligned}
$$

The rest of the proof is similar to the above case $(I)$, so we omit the details.

Thus, the proof of the theorem is completed.

If the number of the non-zero terms of the $p$-adic expansions of $n$ is greater than 2 , there are many types of theorems corresponding to theorem 4 . For example, we obtain the following result.

Theorem 4'. Let $p$ be a prime with $p \geqq 5$. Assume that either of the conditions $\left(I^{\prime}\right)$ and $\left(I I^{\prime}\right)$ below is satisfied.

$\left(I^{\prime}\right) \quad m$ is an integer with $m>2 ; \alpha_{i}(i=1,2, \cdots, m)$ are even integers such that $0<\alpha_{i} \leqq(2 p-2) / 3$; and $k_{i}(i=1,2, \cdots, m)$ are integers such that $k_{m}>k_{m-1}>\cdots>k_{2}>k_{1} \geqq 0$.

(II') $m$ is an even integer with $m>2 ; \alpha_{i}(i=1,2, \cdots, m)$ are odd integers such that $0<\alpha_{i} \leqq(2 p-1) / 3$ if $i$ is even and $0<\alpha_{i} \leqq(p-2) / 3$ if $i$ is odd ; and $k_{i}(i=1,2, \cdots, m)$ are integers such that $k_{m}>k_{m-1}>$ $\cdots>k_{2}>k_{1} \geqq 0$. 
Then $L^{n}(p) \nsubseteq R^{3 n+1} \quad$ for $n=\sum_{i=1}^{m} \alpha_{i} p^{k i}$.

The proof is quite similar to that of Theorem 4, so we shall omit the proof.

Theorem 5. Let $p$ be an odd prime. Assume that either of the conditions (III) and (IV) below is satisfied.

(III) $\alpha$ is an even integer with $0 \leqq \alpha \leqq(2 p-2) / 3, \beta$ is an odd integer with $0<\beta \leqq(2 p-1) / 3$, and $l$ and $k$ are integers such that $l>k \geqq 0$ and $l>1$ if $\alpha>0$, or $k>1$ if $\alpha=0$.

(IV) $\alpha$ is an odd integer with $0<\alpha \leqq(2 p-1) / 3, \beta$ is an even integer with $0 \leqq \beta \leqq(p-1) / 3$, and $l$ and $k$ are integers such that $l>k>0 ; l>1$ if $k=0$ and $\alpha>1$; or $l>2$ if $k=0$ and $\alpha=1$.

Then $L^{n}(p) \nsubseteq R^{3 n}$ for $n=\alpha p^{l}+\beta p^{k}$.

We are indebted to Professor Y. Saito for the proof of the theorem.

Proof. First, we consider the case when the condition (III) is satisfied. Suppose that $L^{n}(p) \nsubseteq R^{3 n}$ for $n=\alpha p^{l}+\beta p^{k}$. Let $\nu$ be an oriented normal bundle of dimension $n-1$. The highest dimensional non-zero Pontrjagin class $\bmod p$ of $\nu$ is

$$
\bar{p}_{(n-1) / 2}=(-1)^{(n-1) / 2}\left(\begin{array}{c}
n+(n-1) / 2 \\
(n-1) / 2
\end{array}\right) x^{n-1}\left(\in H^{2 n-2}\left(L^{n}(p) ; Z_{p}\right)\right),
$$

where $x$ is a generator of $H^{2}\left(L^{n}(p) ; Z_{p}\right)$, since

$$
\begin{aligned}
\left(\begin{array}{c}
n+(n-1) / 2 \\
(n-1) / 2
\end{array}\right) & =\left(\begin{array}{l}
\frac{3 \alpha}{2} p^{l}+\frac{3 \beta-1}{2} p^{k}+\frac{p-1}{2} p^{k-1}+\cdots+\frac{p-1}{2} p+\frac{p-1}{2} \\
\frac{\alpha}{2}-p^{i}+\frac{\beta-1}{2} p^{k}+\frac{p-1}{2} p^{k-1}+\cdots+\frac{p-1}{2} p+\frac{p-1}{2}
\end{array}\right) \\
& \equiv\left(\begin{array}{c}
\frac{3 \alpha}{2} \\
\frac{\alpha}{2}
\end{array}\right) \cdot\left(\begin{array}{c}
\frac{3 \beta-1}{2} \\
\frac{\beta-1}{2}
\end{array}\right) \neq 0(\bmod p) .
\end{aligned}
$$

Let $\bar{\chi}\left(\in H^{n-1}\left(L^{n}(p) ; Z_{p}\right)\right)$ be the Euler class $\bmod p$ of $\nu$. Since $\bar{\chi}^{2}=\bar{p}_{(n-1) / 2}$, we have $\bar{\chi} \neq 0$. Let $s$ and $r$ be integers given by the equation:

$$
\alpha p^{\prime}+\beta p^{k}=s(p-1)+r \quad(0 \leqq r<p-1),
$$


and let $v(=s+\varepsilon)$ be $s$ or $s+1$ according as $[r / 2]=0$ or $[r / 2] \geqq 1$ respectively. Denote by $a$ an integer with $2 a p^{\prime \prime}>4 n+3$.

Now, by Theorem 3 , there is a map

$$
g: S^{t}\left(L^{n}(p) / L^{(n-3) / 2}(p)\right) \rightarrow L^{a p^{n}-(n+1) / 2}(p) / L^{a p^{t}-n-2}(p)
$$

which induces isomorphisms of all cohomology groups with $Z_{p}$ coefficients, where $t$ is a positive integer given by

$$
t=2 a p^{v}-3 n-1 \text {. }
$$

Let

$$
E^{t}: H^{q-t}\left(L^{n}(p) / L^{(n-3)^{\prime 2}}(p) ; Z_{p}\right) \approx H^{q}\left(S^{t}\left(L^{n}(p) / L^{(n-3 / 2 / 2}(p)\right) ; Z_{p}\right)
$$

be the $t$-fold suspension isomorphism and let $\left(E^{t}\right)^{-1} \circ g^{*}=G$. Since $E$ and $g^{*}$ commute with the Steenrod reduced power operation respectively, so is $G$.

If $k>0$, we define a positive integer $q$ by the equation:

$$
q=2 a p^{\prime \prime}-n-p^{2},
$$

and consider the following commutative diagram.

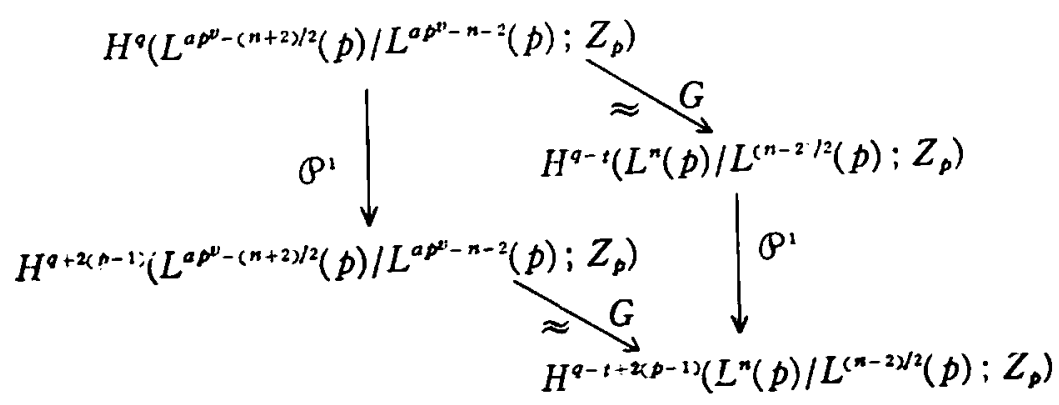

As in the proof of Theorem 4, we can show that $P^{1}$ on the left is trivial and that $P^{1}$ on the right is non-trivial. This is a contradiction.

If $k=0$, we can give the proof by defining $q=2 a p^{v}-n-2 p^{2}-1$ and using reduced power operations $P^{p}$, similarly in the proof of Theorem 4. We omit the details here.

Secondly, we consider the case when the condition $(I V)$ is satisfied. Suppose that $L^{n}(p) \subseteq R^{3 n}$ for $n=\alpha p^{l}+\beta p^{k}$. Let $\nu$ be an oriented normal bundle of dimension $n-1$. The highest dimen. 
sional non-zero Pontrjagin class $\bmod p$ of $\nu$ is $\bar{p}_{(n-1) / 2}\left(\in H^{2 n-2}\right.$ $\left.\left(L^{n}(p) ; Z_{p}\right)\right)$, because

$$
\begin{aligned}
& \left(\begin{array}{c}
n+(n-1) / 2 \\
(n-1) / 2
\end{array}\right)= \\
& \left(\begin{array}{c}
\frac{3 \alpha-1}{2} p^{k}+\frac{p-1}{2} p^{\prime-1}+\cdots+\frac{p-1}{2} p^{k+1}+\frac{3 \beta+p-1}{2} p^{k}+\frac{p-1}{2} p^{k-1}+\cdots+\frac{p-1}{2} \\
\frac{\alpha-1}{2} p^{\prime}+\frac{p-1}{2} p^{\prime-1}+\cdots+\frac{p-1}{2} p^{k+1}+\frac{\beta+p-1}{2} p^{k}+\frac{p-1}{2} p^{k-1}+\cdots+\frac{p-1}{2}
\end{array}\right) \\
& \equiv\left(\begin{array}{c}
\frac{3 \alpha-1}{2} \\
\frac{\alpha-1}{2}
\end{array}\right) \cdot\left(\begin{array}{c}
\frac{3 \beta+p-1}{2} \\
\frac{\beta+p-1}{2}
\end{array}\right) \neq 0 \quad(\bmod p) .
\end{aligned}
$$

The rest of the proof is similar to the above case $(I I I)$, so we omit the details.

Thus the proof of the theorem is completed.

As an example of a corresponding result to Theorem 5 in case when the number of non-zero terms of the $p$-adic expansion of $n$ is greater than 2 , we have the following

Theorem 5'. Let $p$ be an odd prime. Assume that the condition $\left(I I I^{\prime}\right)$ belou' is satisfied.

$\left(I I I^{\prime}\right) \quad m$ is an integer with $m>2 ; r$ is an integer with $1 \leqq r \leqq m$; $\alpha_{i}(i=1,2, \cdots, r-1)$ are even integers with $0<\alpha_{i} \leqq(p-1) / 3, \alpha_{r}$ is an odd integer with $0<\alpha_{r} \leqq(2 p-1) / 3$ and $\alpha_{i}(i=r+1, \cdots, m)$ are even integers with $0<\alpha_{i} \leqq(2 p-2) / 3$; and $k_{i}(i=1,2, \cdots, m)$ are integers such that $k_{m}>k_{m-1}>\cdots>k_{2}>k_{1} \geqq 0$.

Then $L^{n}(p) \nsubseteq R^{3 n}$ for $n=\sum_{i=1}^{\infty} a_{i} p^{k_{i}}$.

We can prove this theorem by the similar way to Theorem 5 , so we omit the proof.

In the end, we shall discuss the exceptional case $p=3$.

Theorem 6. Let $k$ be a positive integer. If $n=3^{k}, L^{n}(3) \nsubseteq R^{3 n}$.

Proof. If $k>1$, the assertion is true by Theorem 5 . We consider the case $k=1$. Suppose that $L^{3}(3) \subseteq R^{9}$. Let $\nu$ be an 
oriented normal bundle of dimension 2. The highest dimensional non-zero Pontrjagin class $\bmod 3$ of $\nu$ is

$$
\bar{p}_{1}=-x^{2}\left(\in H^{4}\left(L^{3}(3) ; Z_{3}\right)\right),
$$

where $x$ is a generator of $H^{2}\left(L^{3}(3) ; Z_{3}\right)$.

Let $\bar{\chi}\left(\in H^{2}\left(L^{3}(3) ; Z_{3}\right)\right)$ be the Euler class $\bmod 3$ of $\nu$. Then we have $\bar{\chi}^{2}=\bar{p}_{1}=-x^{2}$. But, this is impossible. Therefore, $L^{3}(3) \nsubseteq R^{9}$.

Theorem 7. Let $l$ and $k$ be integers with $l-1>k \geqq 0$. If $n=3^{l}+3^{k}, L^{n}(3) \nsubseteq R^{3 n-1}$.

Proof. Suppose that $L^{n}(3) \subseteq R^{3 n-1}$. Let $\nu$ be an oriented normal bundle of dimension $n-2$. The highest dimensional nonzero Pontrjagin class $\bmod 3$ of $\nu$ is

$$
\bar{p}_{(n-2) / 2}=(-1)^{n / 2} x^{n-2}\left(\in H^{2 n-4}\left(L^{n}(3) ; Z_{3}\right)\right)
$$

because

$$
\left(\begin{array}{c}
n+n / 2 \\
n / 2
\end{array}\right) \equiv 0 \quad(\bmod 3) \quad \text { and } \quad\left(\begin{array}{c}
n+(n-2) / 2 \\
(n-2) / 2
\end{array}\right) \equiv-1 \quad(\bmod 3) .
$$

Let $\bar{\chi}\left(\in H^{n-2}\left(L^{n}(3) ; Z_{3}\right)\right)$ be the Euler class $\bmod 3$ of $\nu$. Since $\bar{\chi}^{2}=\bar{p}_{(n-2) / 2}$, we get $\bar{\chi} \neq 0$. Note that $\varepsilon=0$ (i.e., $v=s$ ) and that $2 \cdot 3^{s}>4 n+3$ for $n \geqq 10$, where $s=n / 2$.

Therefore, by Theorem 3 , there is a map

$$
g: S^{t}\left(L^{n}(3) / L^{(n-4) / 2}(3)\right) \rightarrow L^{3 s-n / 2}(3) / L^{3 s-n-2}(3)
$$

which induces isomorphisms of all cohomology groups with $Z_{3}$ coefficients, where $t$ is a positive integer given by

$$
t=2 \cdot 3^{s}-3 n \text {. }
$$

Let

$$
E^{t}: H^{q-t}\left(L^{n}(3) / L^{(n-t) / 2}(3) ; Z_{3}\right) \approx H^{q}\left(S^{t}\left(L^{n}(3) / L^{(n-4) / 2}(3)\right) ; Z_{3}\right)
$$

be the $t$-fold suspension isomorphism and let $\left(E^{t}\right)^{-1} \circ g^{*}=G$. Since $E$ and $g^{*}$ commute with the reduced power operations respectively, so is $G$.

Define a positive integer $q$ by the equation:

$$
q=2 \cdot 3^{s}-n-2 \cdot 3^{k+2}
$$


and consider the following commutative diagram, where the two $P^{3^{k+1}}$ are $3^{k+1}$-th Steenrod reduced power operations $\bmod 3$.

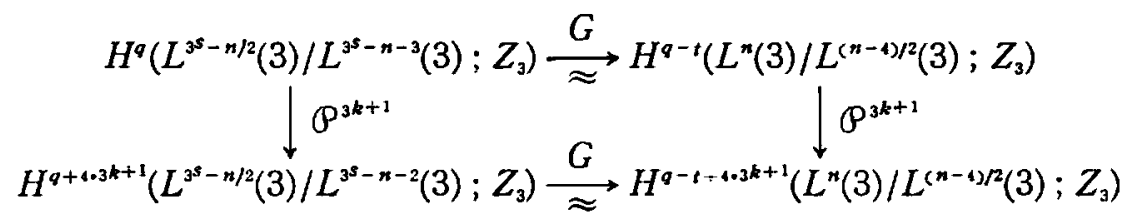

If $l>k+2$,

$$
q-\left\{2\left(3^{s}-n-2\right)+1\right\}=3^{l}+3^{k}-2 \cdot 3^{k+2}+3 \geqq 13 .
$$

Hence, we see that the two operations $P^{3 k+1}$ on the left and on the right in the diagram are equivalent to the following two operations respectively :

$$
\begin{aligned}
& \mathcal{P}^{3 k+1}: H^{q}\left(L^{3 s-n / 2}(3) ; Z_{3}\right) \rightarrow H^{q+4.33^{k+1}}\left(L^{3 s-n / 2}(3) ; Z_{3}\right), \\
& \mathcal{P}^{3^{k+1}}: H^{q-1}\left(L^{n}(3) ; Z_{3}\right) \rightarrow H^{q-\left\{+4.33^{k+1}\right.}\left(L^{n}(3) ; Z_{3}\right) .
\end{aligned}
$$

For a generator $x^{q / 2}$ of $H^{q}\left(L^{3 s-n / 2}(3) ; Z_{3}\right)$ we have

$$
\mathbb{P}^{3 k+1} x^{q / 2}=\left(\begin{array}{l}
q / 2 \\
3^{k+1}
\end{array}\right) x^{q / 2+2 \cdot 3^{k+1}}=x^{q / 2+2 \cdot 3^{k+1}} \neq 0,
$$

since $q / 2=2 \cdot 3^{s-1}+\cdots+2 \cdot 3^{t}+3^{t-1}+\cdots+3^{k+3}+3^{k+1}+3^{k}$. On the other hand, for a generator $x^{(q-t) / 2}$ of $H^{q-t}\left(L^{n}(3) ; Z_{3}\right)$ we have

$$
\mathcal{P}^{3 k+1} x^{(q-t))^{2}}=\left(\begin{array}{c}
(q-t) / 2 \\
3^{k+1}
\end{array}\right) x^{(q-t) / 2+2 \cdot 3 k+1}=0,
$$

since $(q-t) / 2=2 \cdot 3^{t-1}+\cdots+2 \cdot 3^{k+2}+3^{k}$.

Therefore, $\beta^{3 k+1}$ on the left in the above diagram is nontrivial, while $\mathcal{P}^{\beta^{k+1}}$ on the right is trivial. This is a contradiction.

If $l=k+2, n / 2=3^{k+1}+2 \cdot 3^{k} \equiv 1(\bmod 2)$. Then we have $\dot{p}_{(n-2) / 2}$ $=-x^{n-2}$. But, this is inconsistent with the fact that $\bar{\chi}^{2}=\bar{p}_{(x-2) / 2}$.

Thus the proof of the theorem is completed.

\section{§5. Remarks}

T. Kambe has proved in [6] the following non-immersion theorem for lens spaces.

Let $p$ be an odd prime. Then $L^{n}(p) \nsubseteq R^{2 n+2 L(n, p)}$, where $L(n, p)$ 
is the integer defined by

$$
L(n, p)=\max \left\{i \leqq[n / 2] \mid\left(\begin{array}{c}
n+i \\
i
\end{array}\right) \neq 0 \bmod p^{i+[(n-2 i) /(p-1)]}\right\}
$$

From this theorem, the following results are obtained.

1) Let $p$ be a prime with $p \geqq 5$. $\alpha$ and $\beta$ are integers defined in (I) or (II), and $l$ and $k$ are integers such that $l>k \geqq 0$.

Then $L^{n}(p) \nsubseteq R^{3 n}$ for $n=\alpha p^{2}+\beta p^{k}$.

2) Let $p$ be an odd prime. $\alpha$ and $\beta$ are integers defined in (III) or (IV), and $l$ and $k$ are integers such that $l>k \geqq 0$.

Then $L^{n}(p) \nsubseteq R^{3 n-1}$ for $n=\alpha p^{l}+\beta p^{k}$.

3) Let $l$ and $k$ be integers with $l \geqq k \geqq 0$.

Then $L^{n}(3) \nsubseteq R^{3 n-2}$ for $n=3^{l}+3^{k}$.

These results 1 ), 2) and 3 ) are also obtained from the well known theorem:

Let $M^{n}$ be an n-dimensional manifold. If $M^{n} \subseteq R^{n+k}$, then $p_{i}\left(M^{n}\right)=0$ except 2-torsions for $i>\left[k_{i}^{\prime}\right]$, where $\dot{p}_{i}\left(M^{n}\right)\left(\in H^{\prime i}\left(M^{n}\right.\right.$; $Z)$ ) is the i-th normal Pontrjagin class.

As for the immersion theorem for lens spaces, recently $F$. Uchida has proved in [13] the following result :

Let $p$ be an odd prime,

1) If $n$ is odd, $L^{n}(p) \subseteq R^{3 n+3}$.

2) If $n$ is even, $L^{n}(p) \subseteq R^{3 n+4}$.

Yoshida College,
Kyoto University

\section{REFERENCES}

[1] J. Adem and S. Gitler, Non-immersion theorems for real projective spaces, Bol. Soc. Mat. Maxicana, 9 (1964), 37.50.

[2] M. F. Atiyah, Thom complexes, Proc. London Math. Soc., 11 (1961), 291-310.

[3] M. F. Atiyah, Immersions and embeddings of manifolds. Topology 1 (1961), 125-132.

[4] M. W. Hirsch, Immersions of manifolds, Trans. Amer. Math. Soc., 93 (1959), 242-276.

[5] I. M. James, On the immersion problem for real projective spaces, Bull. Amer. Math. Soc., 69 (1963), 231-238.

[6] T. Kambe, The structure of $K_{\mathrm{\Lambda}}$-rings of the lens space and their applications, J. Math. Soc. Japan, 18 (1966), 135-146.

[7] T. Kambe, H. Matsunaga and H. Toda, A note on stunted lens space, J. Math. Kyoto Univ., 5 (1966), 143-149. 
[8] J. Milnor, Lectures on characteristic elasses, (mimeographed notes), Princeton University, 1957.

[9] R. Nakagawa and T. Kobayashi, Non-embeddability of lens spaces mod 3, J. Math. Kyoto Univ, 5 (1966), 313-324.

[10] B. J. Sanderson, Immersions and embeddings of projective spaces, Proc. London Math. Soc., 14 (1964), 137-153.

[11] N. E. Steenrod, Cohomology Operations, Princeton University Press, 1962.

[12] R. Thom, Espaces fibrés en sphères et carrés de Steenrod, Ann. Sci. Ecole Norm. Sup., 69 (1952), 109-182.

[13] F. Uchida, Immersions of lens spaces. 